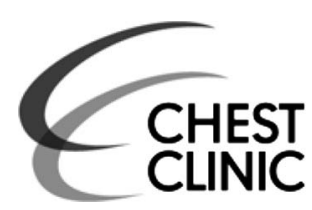

\title{
Mortality reduction in adult community-acquired pneumonia in the UK (2009-2014): results from the British Thoracic Society audit programme
}

\author{
Priya Daniel, ${ }^{1}$ Mark Woodhead ${ }^{2}$ Sally Welham, ${ }^{3}$ Tricia M Mckeever, ${ }^{4}$ Wei Shen Lim, ${ }^{1}$ \\ on behalf of the British Thoracic Society
}

- Additional material is published online only. To view please visit the journal online (http://dx.doi.org/10.1136/ thoraxjnl-2016-208937).

${ }^{1}$ Department of Respiratory Medicine, Nottingham University Hospitals NHS Trust, Nottingham, UK

${ }^{2}$ Department of Respiratory Medicine, Central Manchester University Hospitals NHS Foundation Trust, Manchester, UK

${ }^{3}$ British Thoracic Society, London, UK

${ }^{4}$ Division of Epidemiology, University of Nottingham, Nottingham, UK

\section{Correspondence to} Dr Priya Daniel, Department of Respiratory Medicine, David Evans Building, City Hospital Campus, Nottingham University Hospitals NHS Trust, Hucknall Road, Nottingham NG5 1PB, UK; priyasosha. daniel@nuh.nhs.uk

Received 19 May 2016 Revised 16 June 2016 Accepted 10 July 2016 Published Online First 18 August 2016

\section{Linked}

- http://dx.doi.org/10.1136/ thoraxinl-2016-209284

CrossMark

To cite: Daniel $P$,

Woodhead M, Welham S,

et al. Thorax

2016;71:1061-1063.

\section{ABSTRACT}

Community-acquired pneumonia (CAP) is a leading cause of death in the UK. In this analysis of 23315 cases from the British Thoracic Society national CAP audit, an overall reduction in 30-day inpatient mortality over 6 years was observed - 2014 compared with 2009 adjusted OR $0.86(95 \% \mathrm{Cl} 0.68$ to 1.08 , p for trend 0.004 ). Significant increases in the proportions of patients who had (a) a chest X-ray and (b) the first antibiotic dose within 4 hours of admission were also observed (3.7\% and $11.5 \%$ increases respectively). Further reductions in mortality may follow the 2016 National Institute for Health and Care Excellence Pneumonia Quality Standard.

\section{INTRODUCTION}

In the UK, national guidelines for the management of community-acquired pneumonia (CAP) have been in place since 2003. One of the expectations of clinical guidelines is to contribute towards improved standards of care and improved clinical outcomes.

In 2014, based on data from the UK Office of National Statistics, there were over 25000 registered deaths from pneumonia making it the sixth-leading cause of death in England and Wales and over the 10-year period (2004-2014) age-standardised death rates from pneumonia fell for both men (837 to 572 per million population) and women (668 to 429 per million population). Studies from the USA have also observed reductions in pneumonia mortality over the past decade. ${ }^{1}$ However, whether these observations represent true reductions in mortality is unclear; alternative explanations may include changes in coding practices over time and changing thresholds for hospital admission with less unwell patients being hospitalised in more recent years.

The British Thoracic Society (BTS) published guidelines for the management of adults with CAP in 2009 and initiated a national BTS CAP audit programme in the same year. In contrast to administrative datasets, the BTS CAP audit captures data from adults with radiologically confirmed CAP, stratified according to pneumonia severity. In this analysis, trends in mortality and key processes of care from 2009 to 2014 were examined.

\section{METHODS}

\section{Study design}

Since 2009, NHS Institutions in England, Scotland, Wales and Northern Ireland have been invited to participate in the national BTS CAP audit for adults hospitalised in acute trusts. The audit was conducted annually from 2009 to 2012 and subsequently in 2014; institutions were required to prospectively or retrospectively identify adults with a primary diagnosis of CAP who were hospitalised during the winter period from 1 December to 31 January. Medical notes and chest radiographs of identified patients were reviewed by investigators at each participating site and entered into the audit if the patients fulfilled the following eligibility criteria: (a) age $\geq 16$ years with new infiltrates on chest radiograph, (b) presence of signs and symptoms of a lower respiratory tract infection, (c) no recent hospital discharge within the preceding 10 days of index admission and (d) who were not immunocompromised. Demographic and clinical data were extracted using a standardised pro-forma and entered onto a secure website. The BTS Professional and Organisational Standards Committee determined that ethical approval was not required for the conduct of this audit.

\section{Statistical considerations}

Statistical analyses were performed using Stata/IC (V.13.1; StataCorp, 2013). Baseline demographics and clinical features of adults with CAP were compared over the audit years. The independent association between the year of admission and 30-day inpatient (IP) mortality was examined using a multivariable logistic regression model, allowing for clusters by institution. The year of admission was included in the multivariable model as both a categorical variable, to obtain the ORs in reference to 2009 , and a continuous variable, to attain the $p$ value for trend. The final model comprised age as a categorical variable, variables from the CURB65 score excluding the age component (Confusion, urea $>7 \mathrm{mmol} / \mathrm{L}$, respiratory rate $\geq 30 / \mathrm{min}$, systolic blood pressure $<90 \mathrm{~mm} \mathrm{Hg}$ or diastolic blood pressure $\leq 60 \mathrm{~mm} \mathrm{Hg}$ ), critical care admission and those comorbid illnesses which were significantly associated with 30-day IP mortality and/or which led to a $>10 \%$ change in the regression coefficient between year of admission and mortality. Changes in categorical variables related to processes of care were compared using the $\chi^{2}$ test of trend.

\section{RESULTS}

Study population

A total of 218 institutions (66 institutions in 2009 , rising to 154 in 2014) submitted data for 24187 
adults over the 6-year period of the audit. Data regarding 30-day IP mortality were unavailable for 204 adults-four patients were aged under 16 years and a further 664 adults were admitted to hospital outside the audit periods; exclusion of these patients left data from 23315 adults for analysis. Data for analysis were obtained for 2610 cases in 2009, 3456 cases in 2010, 5282 cases in 2011, 5499 cases in 2012 and 6468 cases in 2014.

\section{Baseline characteristics}

The median age of the study population was 77 years (IQR 6285 years) and the overall 30-day IP mortality was $18.0 \%$ $(n=4198)$. There was a gradual increase in the median age of hospitalised adults over the 6 years of the audit $(75$ years in 2009 vs 78 years in 2014, $\mathrm{p}<0.001$ ). The frequencies of comorbid illnesses varied across the years (see online supplementary table S1). There was an increase in the proportion of low severity CAP (according to CURB65 score) in 2010 compared with other years and an overall decrease in critical care admissions from 2009 to $2014(7.0 \%$ in 2009 vs $5.1 \%$ in 2014 , p for trend <0.001).

\section{Mortality}

Crude 30-day IP mortality was 18.4\%, 19.5\%, 18.4\%, 17.4\% and $17.3 \%$ in 2009, 2010, 2011, 2012 and 2014, respectively. There was a significant reduction in the adjusted OR (AOR) for mortality from 2009 (reference) to 2014 (AOR 0.86 (95\% CI 0.68 to 1.08$)$ : $\mathrm{p}$ for trend $=0.004$ ) (figure 1 ).

\section{Processes of care}

Admissions through the emergency department increased significantly from 2009 to $2014(67.2 \%$ vs $76.3 \%$, p for trend $<0.001)$. There was a significant increase in the proportion of patients who received their first antibiotic dose within 4 hours of admission: $57.0 \%, 57.0 \%, 60.8 \%, 64.5 \%$ and $68.5 \%$ in 2009, 2010, 2011, 2012 and 2014, respectively ( $p$ for trend $<0.001)$. The proportions of patients who had a chest X-ray (CXR) within 4 hours of admission were 76.5\%, 75.5\%, $80.9 \%, 80.6 \%$ and $80.2 \%$ over the years 2009-2014 (p for trend $=0.055$ ). Adherence to local antibiotic guidelines increased from $55.6 \%$ in 2009 to $57.3 \%$ in 2014 ( $\mathrm{p}$ for trend=0.035); however, patients with moderate-severity and high-severity

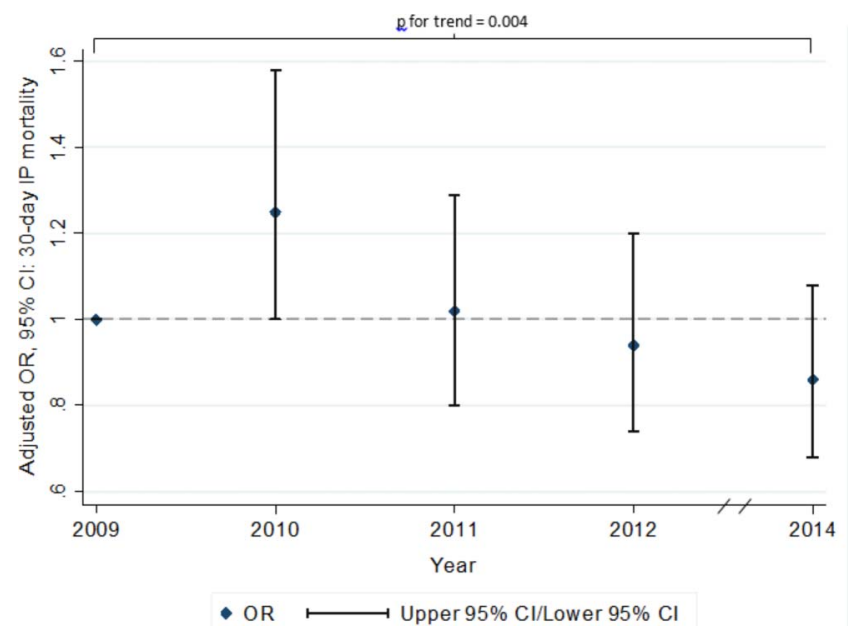

Figure 1 Adjusted 30-day inpatient mortality between 2009 and 2014 among patients admitted to hospital with a primary diagnosis of community-acquired pneumonia is shown. disease (according to CURB65 score) were less likely to receive combination $\beta$-lactam and macrolide therapy over this period $(51.9 \%$ vs $48.0 \%$, p for trend $<0.001)$.

\section{DISCUSSION}

The main finding of this study is that in the UK, over a 6-year period from 2009 to 2014, there has been a 14\% decrease in adjusted 30-day IP mortality in adults hospitalised with CAP. This has occurred without change in pneumonia severity on admission and despite an increase in median age. Over the same period, there have been improvements in some processes of care measures, specifically a significant $11.5 \%$ increase in the proportion of adults who received their first antibiotic dose within 4 hours of admission, $3.7 \%$ increase in the proportion of adults who had a CXR within 4 hours of admission and 1.7\% increase in guideline-adherent antibiotic therapy. Some of these improvements in processes of care may be, at least partially, related to the significant $9.1 \%$ increase in the proportion of cases admitted through the emergency department.

To our knowledge, this is the largest UK study of temporal mortality trends in adults hospitalised with CAP. The major strengths of this analysis are the size of the dataset, the inclusion of 'real-world' cases from multiple institutions with radiologically confirmed CAP and the availability of detailed data enabling adjustment for case-mix and disease severity. Possible explanations for the observed reductions in mortality include the associated improvements in the early (within 4 hours of admission) diagnosis and antibiotic treatment of patients. ${ }^{2}$ Other healthcare interventions implemented by local institutions as part of the audit cycle and which have not been measured by this audit may also be important. Finally, a Hawthorne effect cannot be excluded though this seems less likely as a single explanation for the findings, given the trend observed over a period of 6 years.

Over the study period, the highest adjusted 30-day IP mortality was observed in winter 2010/2011; this coincides with the 'third wave' of the 2009 H1N1 influenza pandemic when higher rates of severe influenza infection, particularly affecting younger patients, with increased influenza-related fatalities were observed nationally. In a sensitivity analysis, excluding data from cases admitted in 2010, the trend in mortality reduction remained significant ( $\mathrm{p}$ for trend $=0.041$, data not shown). Our results are in keeping with the mortality trends described in a Spanish cohort of 4558 adults with CAP hospitalised over a 19 -year period. ${ }^{3}$ In that study, similar increases in the proportion of patients receiving first dose antibiotic within 4 hours of admission were reported over the study periods.

Overall, this study suggests there has been a true reduction in pneumonia mortality over the past 6 years although a causal association with adherence to national CAP guidelines cannot be established. Similar to recommendations made in the 2009 BTS CAP Guideline, the 2016 National Institute for Health and Care Excellence Pneumonia Quality Standard advocates the following: ${ }^{4}$

1. Adults with suspected CAP in hospital have a CXR and receive a diagnosis within 4 hours of presentation

2. Adults have a mortality risk assessment using the CURB65 score when they are diagnosed with CAP in hospital

3. Adults with CAP who are admitted to hospital start antibiotic therapy within 4 hours of presentation.

Further study is required to determine levels of adherence to this standard and whether adherence is associated with variation in pneumonia mortality. 
Acknowledgements On behalf of the British Thoracic Society (BTS), the authors thank all the clinicians and their teams who participated in the BTS

Community-acquired Pneumonia (CAP) audit and who drove improvement projects at their hospitals. They also acknowledge the indomitable support of the BTS Audit team.

Contributors WSL and SW were instrumental in organising the BTS national CAP audit; PD and TMM analysed and interpreted the data and drafted the article; TMM provided statistical input; TMM, MW, SW and WSL critically evaluated the article; all authors revised and reviewed the article prior to final approval.

Competing interests All authors have completed the International Committee of Medical Journal Editors uniform disclosure form. PD has received salaries funded by an unrestricted grant from Pfizer. WSL has received unrestricted investigator-initiated research funding from Pfizer for unrelated research in pneumonia. MW reports speaking at National and International Scientific meetings which were supported by multiple pharmaceutical companies but without personally receiving direct funding from any individual pharmaceutical companies.
Provenance and peer review Not commissioned; externally peer reviewed.

\section{REFERENCES}

1 Ruhnke GW, Coca Perraillon M, Cutler DM. Mortality reduction among pneumonia patients still substantial despite the impact of coding changes. Am J Med 2013;126:266-9

2 Daniel P, Rodrigo C, Mckeever TM, et al. Time to first antibiotic and mortality in adults hospitalised with community-acquired pneumonia: a matched-propensity analysis. Thorax 2016;71:568-70.

3 Simonetti AF, Garcia-Vidal C, Viasus D, et al. Declining mortality among hospitalized patients with community-acquired pneumonia. Clin Microbiol Infect 2016;22:567.e1-7.

4 Lim WS, Smith DL, Wise MP, et al. British Thoracic Society community acquired pneumonia guideline and the NICE pneumonia guideline: how they fit together. Thorax 2015;70:698-700.

5 National Institute for Health and Care Excellence. Pneumonia in adults. Quality standard (QS110), 2016. 